\title{
First-principles T-matrix calculations of double-ionization energy spectra of atoms and molecules
}

\author{
Yoshifumi Noguchi, Yohei Kudo, Soh Ishii, and Kaoru Ohno ${ }^{\text {a) }}$ \\ Department of Physics, Graduate School of Engineering, Yokohama National University, 79-5 Tokiwadai, \\ Hodogaya, Yokohama 240-8501, Japan
}

(Received 28 July 2005; accepted 23 August 2005; published online 12 October 2005)

\begin{abstract}
Strong electron correlation plays an important role in the determination of double ionization energy, which is required for removing or adding two electrons, particularly in small-sized systems. Starting from the state-of-the-art $G W$ approximation, we evaluate the particle-particle ladder diagrams up to the infinite order by solving the Bethe-Salpeter equation of the $T$-matrix theory to calculate the double-ionization energy spectra of atoms and molecules $\left(\mathrm{Be}, \mathrm{Mg}, \mathrm{Ca}, \mathrm{Ne}, \mathrm{Ar}, \mathrm{Kr}, \mathrm{CO}, \mathrm{C}_{2} \mathrm{H}_{2}, \mathrm{Li}_{2}\right.$, $\mathrm{Na}_{2}$, and $\mathrm{K}_{2}$ ) from first principles. The ladder diagrams up to the infinite order are significant to calculations of double-ionization energy spectra. The present results are in good agreement with available experimental data as well as the previous calculations using, e.g., the configuration-interaction method. (C) 2005 American Institute of Physics. [DOI: 10.1063/1.2069907]
\end{abstract}

\section{INTRODUCTION}

In electronic systems, two-particle excitations that are related to the strong electron correlation between two particles are interesting phenomena. In particular, the doubleionization energy (double electron affinity), which is defined as the energy required for removing (adding) two electrons from (to) a neutral system, reflects the strong Coulomb interaction between holes (electrons). During the last few decades a lot of theoretical attempts were performed to calculate the two-particle excitation spectra. For example, the double electron affinities of small-sized molecules were calculated by using the delta self-consistent-field and configurationinteraction methods, ${ }^{1-4}$ while the double-ionization energies (DIEs) were evaluated from the potential-energy curves of the dications using the multireference configurationinteraction method.,

Another method to calculate two-particle excitation spectra is the two-particle Green's function method of which merit is that one can determine the whole spectra all at once in a single calculation in contrast to the multireference configuration-interaction method. Tarantelli and co-workers formulated a two-particle Green's function method known as algebraic diagrammatic construction $[\mathrm{ADC}(2)$ or $\mathrm{ADC}(3)]$ (Refs. 7-9) which is based on second- or third-order diagrammatic perturbation expansion, calculating the DIEs of various molecules. ${ }^{10-14}$ However, the present approach, which is completely different from the $\operatorname{ADC}(n)$, sums up special kinds of diagrams (e.g., ring or ladder diagrams) up to the infinite order. To calculate the DIE spectra, the ladder diagrams up to the infinite order that accurately give the short-range repulsive Coulomb interactions between two holes are significant because the short-range repulsive Coulomb interactions dominantly affect the DIE spectra particularly in the small-sized systems.

Recently, the first-principles Green's function methods

${ }^{\text {a)} E l e c t r o n i c ~ m a i l: ~ o h n o @ y n u . a c . j p ~}$ that sum up special kinds of diagrams up to the infinite order were successfully applied to the excited-state physics. Oneparticle ionization energy spectra can be accurately evaluated as the poles of the one-particle Green's function by the stateof-the-art $G W$ calculations. ${ }^{15,16}$ Moreover, optical-absorption spectra can be calculated by starting from the $G W$ approximation $^{17}$ (GWA) and solving the Bethe-Salpeter equation (BSE) for the electron-hole Green's function. ${ }^{18-23}$ In these methods, the physics such as the electronic screening and the exciton binding can be treated more transparently.

The spectra of DIEs can be calculated from first principles as the poles of the two-particle Green's function in a manner analogous to calculations of the optical-absorption spectra using the BSE. However, there has been no such theoretical attempt so far. Only one theoretical calculation was carried out by Springer et al. ${ }^{24}$ who used the $T$-matrix theory for the electron-electron and hole-hole Green's functions to calculate the imaginary part of the one-particle selfenergy. In the present paper, we will show for the first time that the spectra of DIEs can be accurately calculated by starting from the GWA, solving the BSE for the electron-electron or hole-hole Green's function in the first-principles $T$-matrix theory successfully.

The key point is that the DIE strongly reflects the effects of the particle-particle interactions in contrast to the first ionization potential (IP) or electron affinity (EA). It is inevitable to treat the multiple scattering between two particles within the ladder approximation. The smaller the system, the stronger the effect of this interaction. This is because the effect of the electron-electron Coulomb interaction becomes quite significant in confined geometries. Here, we show that the present method can well reproduce the experimental values of the DIE spectra of atoms (Be, $\mathrm{Mg}, \mathrm{Ca}, \mathrm{Ne}, \mathrm{Ar}$, and $\mathrm{Kr}$ ) and molecules $\left(\mathrm{Li}_{2}, \mathrm{Na}_{2}, \mathrm{~K}_{2}, \mathrm{CO}\right.$, and $\left.\mathrm{C}_{2} \mathrm{H}_{2}\right)$. 

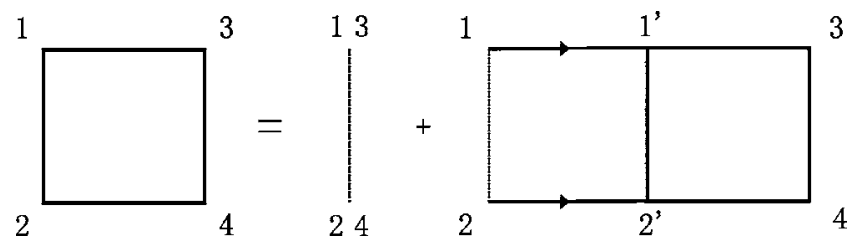

FIG. 1. Schematic representation of the BSE for $T$ matrix (square). The dotted line represents the bare Coulomb interaction $U$. The solid lines with arrows represent the one-particle Green's function $G$.

\section{METHODOLOGY}

We employ an all-electron mixed basis approach, ${ }^{25-27}$ in which both atomic orbitals (AOs) and plane waves (PWs) are used as basis-set functions. The advantage of this approach is that one can express not only spatially localized states but also extended states such as free electron states above the vacuum level. After the electronic states are treated within the local density approximation (LDA) of the densityfunctional theory, we carry out the calculations using the GWA and then proceed to the $T$-matrix theory.

Single quasiparticle energies such as the IP and EA are obtained by solving the Dyson's equation: $G=G^{0}+G^{0} \Sigma G$, where $\Sigma$ is the electron self-energy operator. Here, we introduce the short-hand notation $1 \equiv\left(\mathbf{r}_{1}, t_{1}\right)$. Within the GWA, the electron self-energy operator is given by $\Sigma^{\mathrm{GWA}}\left(1,1^{\prime}\right)$ $=i G\left(1,1^{\prime}\right) W\left(1^{+}, 1^{\prime}\right)$, which is divided into the exchange part $\Sigma_{x}$ and the rest. Usually, the dynamically screened Coulomb interaction $W$ is evaluated within the random-phase approximation (RPA). In diagrammatic sense, the RPA is equivalent to summing ring diagrams up to the infinite order. In the present study, we use the generalized plasmon-pole model, ${ }^{15}$ giving reliable one-particle exitation energies. ${ }^{28}$

In order to evaluate the DIE, we introduce the twoparticle Green's function and $T$ matrix, which describes the multiple scattering between two particles ${ }^{29}$ and the Coulomb hole. The $T$ matrix satisfies the following BSE: ${ }^{24}$

$$
\begin{aligned}
T(1,2 \mid 3,4)= & U(1,2) \delta(1-3) \delta(2-4) \\
& +i U(1,2) \int d 1^{\prime} d 2^{\prime} K\left(1,2 \mid 1^{\prime}, 2^{\prime}\right) T\left(1^{\prime}, 2^{\prime} \mid 3,4\right),
\end{aligned}
$$

where $U\left(\mathbf{r}_{1}, \mathbf{r}_{2}\right) \delta\left(t_{1}-t_{2}\right)$ denotes the bare Coulomb interaction. The schematic representation of this equation is shown in Fig. 1. Here, $K$ represents the disconnected part of the two-particle Green's function defined by $K\left(1,2 \mid 1^{\prime}, 2^{\prime}\right)$ $=i G\left(1^{\prime}, 1\right) G\left(2^{\prime}, 2\right)$. The Fourier transformation of this function is given by

$$
\begin{aligned}
K\left(\mathbf{r}_{1} \mathbf{r}_{2} \mid \mathbf{r}_{1}^{\prime} \mathbf{r}_{2}^{\prime} ; \omega\right)= & -\sum_{\nu \mu} \frac{\psi_{\nu}^{*}\left(\mathbf{r}_{1}\right) \psi_{\nu}\left(\mathbf{r}_{1}^{\prime}\right) \psi_{\mu}\left(\mathbf{r}_{2}^{\prime}\right) \psi_{2}^{*}\left(\mathbf{r}_{2}\right)}{\omega-E_{\nu}-E_{\mu}-i \eta} \\
& +\sum_{\nu \mu}^{\text {emp }} \frac{\psi_{\nu}\left(\mathbf{r}_{1}^{\prime}\right) \psi_{\nu}^{*}\left(\mathbf{r}_{1}\right) \psi_{\mu}^{*}\left(\mathbf{r}_{2}\right) \psi_{\mu}\left(\mathbf{r}_{2}^{\prime}\right)}{\omega-E_{\nu}-E_{\mu}+i \eta},
\end{aligned}
$$

where $E_{\nu}$ is the GWA quasiparticle energy and $\eta$ is a positive infinitesimal number.

We use the LDA wave functions $\psi_{\nu}(\mathbf{r})$ instead of the true excited wave functions throughout the calculation because they differ negligibly except when $E_{\nu}$ approaches the vacuum level. ${ }^{30}$ The BSE (1) can be rewritten by introducing matrix elements sandwiched by the LDA wave functions as follows:

$$
\sum_{\nu \mu}\left(\frac{\delta_{\alpha \nu} \delta_{\beta \mu}}{f_{\nu \mu} K_{\nu \mu}}-U_{\alpha \beta \nu \mu} f_{\nu \mu}\right) f_{\nu \mu} K_{\nu \mu} T_{\nu \mu \gamma \delta}=U_{\alpha \beta \gamma \delta},
$$

where we define $f_{\nu \mu}$ as $f_{\nu \mu}=-\delta_{\nu}^{\text {occ }} \delta_{\mu}^{\text {occ }}+\delta_{\nu}^{\text {emp }} \delta_{\mu}^{\text {emp }}$, and $\delta_{\nu}^{\text {occ }}\left(\delta_{\nu}^{\text {emp }}\right)$ is equal to unity if $\nu$ is occupied (empty) and zero otherwise. We solve the eigenvalue problem as follows:

$$
\sum_{\nu \mu} H_{\alpha \beta \nu \mu} A_{\nu \mu}(\Omega)=\Omega A_{\alpha \beta}(\Omega),
$$

where, $H_{\alpha \beta \nu \mu}$ are the two-particle Hamiltonian matrix elements that are independent of $\omega$,

$$
H_{\alpha \beta \nu \mu} \equiv\left(\frac{1}{f_{\nu \mu} K_{\nu \mu}}-\omega\right) \delta_{\alpha \nu} \delta_{\beta \mu}-U_{\alpha \beta \nu \mu} f_{\nu \mu} .
$$

From the eigenvalues $\Omega$ and eigenfunctions $A_{\nu \mu}(\Omega)$, one can construct the $T$ matrix associated with both the hole-hole and electron-electron Green's functions. The spectra of eigenvalues $\Omega$, which are the poles of the $T$ matrix, directly provide the DIE spectra. The present $T$ matrix treats accurately the short-range part of the Coulomb interaction ${ }^{29,31}$ that is quite significant in the determination of the DIE. If we neglect the interaction between the two particles, the DIE spectra simply become the sum of two one-particle energies, $E_{\nu}+E_{\mu}$, obtained from the GWA.

We employ the supercell approximation that successfully gives wave functions and quasiparticle energies. ${ }^{26,27,32}$ For the calculation of isolated $\mathrm{Be}$ and $\mathrm{Mg}$ atoms, we employ a fcc supercell with a cubic edge of 35 a.u. and PW cutoff energy of $6.5 \mathrm{Ry}$; for $\mathrm{Ca}$, a slightly larger supercell of edge of 40 a.u. and cutoff energy of 4.9 Ry; a supercell of edge of 18.3, 21.3, and 24.6 a.u. and cutoff energy of 17.0, 12.5, and 6.5 Ry for $\mathrm{Ne}, \mathrm{Ar}$, and $\mathrm{Kr}$, respectively; and for $\mathrm{Li}_{2}, \mathrm{Na}_{2}$, and $\mathrm{K}_{2}$, an even larger supercell of edge of 50 a.u. and PW cutoff energy of 3.1 Ry. In addition, we use 18 and 22 a.u. supercells with PW cutoff energies of 34 and 15.9 Ry for CO and $\mathrm{C}_{2} \mathrm{H}_{2}$, respectively. In the calculation of $\Sigma_{x}$ and $U$, the cutoff energies of $\mathbf{G}$ vectors are $26 \mathrm{Ry}$ for $\mathrm{Be}$ and $\mathrm{Mg}, 19.7 \mathrm{Ry}$ for $\mathrm{Ca}$, 17.0 Ry for Ne, 12.5 Ry for Ar, 6.5 Ry for Kr, 12.5 Ry for alkali-metal diatomic molecules, $117 \mathrm{Ry}$ for $\mathrm{CO}$, and $39.2 \mathrm{Ry}$ for $\mathrm{C}_{2} \mathrm{H}_{2}$. All these values are necessary in order to obtain a good convergence of the results within the error bar of $0.1 \mathrm{eV}$ at the GWA level. The other details of the calculation in the GWA are the same as in Refs. 26, 27, and 32.

\section{RESULTS AND DISCUSSION}

\section{A. Two-valence-electron systems}

First of all, we calculate small two-valence-electron systems ( $\mathrm{Be}, \mathrm{Mg}, \mathrm{Ca}, \mathrm{Li}_{2}, \mathrm{Na}_{2}$, and $\mathrm{K}_{2}$ ). The calculated IPs and DIEs of atoms $(\mathrm{Be}, \mathrm{Mg}$, and $\mathrm{Ca})$ and molecules $\left(\mathrm{Li}_{2}, \mathrm{Na}_{2}\right.$, and $\mathrm{K}_{2}$ ) are presented in Table I together with available experimental data. ${ }^{33,34}$ To our knowledge, there is no experimental result for DIE of $\mathrm{Li}_{2}, \mathrm{Na}_{2}$, and $\mathrm{K}_{2}$. In the column "first ionization potential," the IP is evaluated either in the 
TABLE I. The calculated IPs within the GWA and the LDA, and the DIEs within the $T$-matrix theory (in eV) of atoms $(\mathrm{Be}, \mathrm{Mg}$, and $\mathrm{Ca})$ and molecules $\left(\mathrm{Li}_{2}, \mathrm{Na}_{2}\right.$, and $\left.\mathrm{K}_{2}\right)$. In the column of the "double-ionization energy," the DIEs in the "GWA $\times 2$ " are twice the IPs within the GWA (which does not include the effect of the Coulomb repulsive interaction); " $T$ matrix" denotes the values evaluated by the ladder diagrams of the bare Coulomb interaction $U$.

\begin{tabular}{cccccccc}
\hline \hline & \multicolumn{3}{c}{$\begin{array}{c}\text { First ionization potential } \\
\text { (energy to remove one electron) }\end{array}$} & & \multicolumn{3}{c}{$\begin{array}{c}\text { Double-ionization energy } \\
\text { (energy to remove two electrons) }\end{array}$} \\
\cline { 2 - 3 } \cline { 7 - 8 } \cline { 6 - 8 } & LDA & GWA & Experiment & & GWA $\times 2$ & $T$ matrix & Experiment \\
\hline $\mathrm{Be}$ & 5.61 & 9.19 & $9.32^{\mathrm{a}}$ & & 18.39 & 27.79 & $27.53^{\mathrm{a}}$ \\
$\mathrm{Mg}$ & 4.79 & 7.62 & $7.64^{\mathrm{a}}$ & & 15.24 & 22.97 & $22.67^{\mathrm{a}}$ \\
$\mathrm{Ca}$ & 3.87 & 5.92 & $6.11^{\mathrm{a}}$ & & 11.84 & 17.82 & $17.98^{\mathrm{a}}$ \\
$\mathrm{Li}_{2}$ & 3.24 & 5.27 & $5.14^{\mathrm{b}}$ & & 10.54 & 16.62 & $\ldots$ \\
$\mathrm{Na}_{2}$ & 3.20 & 4.90 & $4.93^{\mathrm{b}}$ & & 9.80 & 15.22 & $\ldots$ \\
$\mathrm{K}_{2}$ & 2.74 & 3.98 & $4.05^{\mathrm{b}}$ & & 7.96 & 12.37 & $\ldots$ \\
\hline \hline
\end{tabular}

${ }^{\mathrm{a}}$ Reference 33 .

${ }^{\mathrm{b}}$ Reference 34.

LDA or in the GWA. The LDA underestimates the experimental values for all atoms and molecules. ${ }^{33,34}$ On the other hand, the agreement between the GWA and the experiments is good (the values for $\mathrm{Li}_{2}, \mathrm{Na}_{2}$, and $\mathrm{K}_{2}$ are already given in Ref. 26). In the column "double-ionization energy," " $T$ matrix" denotes the values evaluated by the ladder diagrams of the bare Coulomb interaction $U$. The column "GWA $\times 2$ " represents the DIEs obtained from the evaluation of only the zeroth order of the two-particle Green's function $K$, not considering the Coulomb repulsive interactions between two created holes.

The difference between the DIEs in the "GWA $\times 2$ " and the experiment is about $9.1 \mathrm{eV}$ for $\mathrm{Be}, 7.4 \mathrm{eV}$ for $\mathrm{Mg}$, and $6.1 \mathrm{eV}$ for $\mathrm{Ca}$. However, these disparities are significantly reduced by taking into account the Coulomb interaction between two holes. The difference between " $T$ matrix" and the experiment becomes about $0.3 \mathrm{eV}$ for $\mathrm{Be}, 0.3 \mathrm{eV}$ for $\mathrm{Mg}$, and $0.2 \mathrm{eV}$ for $\mathrm{Ca}$.

The largest contribution to the DIE of the two-valenceelectron systems is an on-site Coulomb interaction $U_{\alpha \alpha \alpha \alpha}$ in Eq. (5) where $\alpha$ is the highest occupied molecular orbital (HOMO) state. The evaluated $U_{\alpha \alpha \alpha \alpha}$ is about $9.6 \mathrm{eV}$ for $\mathrm{Be}$, $7.9 \mathrm{eV}$ for $\mathrm{Mg}$, and $6.2 \mathrm{eV}$ for $\mathrm{Ca}$. This value tends to be smaller as the system size increases because the valence orbital tends to extend further. The same is true for the $U_{\alpha \alpha \alpha \alpha}$ of $\mathrm{Li}_{2}, \mathrm{Na}_{2}$, and $\mathrm{K}_{2}$, being about $6.3,5.6$, and $4.6 \mathrm{eV}$, respectively.

\section{B. Rare-gas atoms}

The calculated IPs of rare-gas atoms $(\mathrm{Ne}, \mathrm{Ar}$, and $\mathrm{Kr})$ are presented in Table II. The values of column "LDA" in Table

TABLE II. The calculated IPs within the GWA and the LDA of Ne, Ar, and $\mathrm{Kr}$ (in $\mathrm{eV}$ ).

\begin{tabular}{lrcc}
\hline \hline & \multicolumn{3}{c}{ First ionization potential } \\
\cline { 2 - 4 } & LDA & GWA & Experiment $^{\mathrm{a}}$ \\
\hline $\mathrm{Ne}$ & 13.54 & 22.28 & 21.56 \\
$\mathrm{Ar}$ & 10.40 & 15.91 & 15.76 \\
$\mathrm{Kr}$ & 9.42 & 14.08 & 14.00 \\
\hline
\end{tabular}

${ }^{\mathrm{a}}$ Reference 33 .
II are considerably smaller than the experimental values, ${ }^{33}$ while the results within the GWA are in good agreement with experiments.

Table III, on the other hand, shows the calculated spectra of DIEs of $\mathrm{Ne}$, Ar, and $\mathrm{Kr}$ and the corresponding experiments. ${ }^{35}$ In every case of rare-gas atoms, " $T$ matrix" considerably improves GWA $\times 2$. The HOMO state of these atoms is threefold degenerated. The spectra of DIEs split into several states such as $\Pi, \Delta$, and $\Sigma$, due to the symmetry of the holes when two arbitrary holes are created from the $p$-type valence orbitals. The three states listed in Table III can be identified from the information of the eigenfunctions (4). However, if we neglect the interaction between two holes, the GWA $\times 2$ would not be able to distinguish these states.

The on-site Coulomb interaction $U_{\alpha \alpha \alpha \alpha}$ is about $28.5 \mathrm{eV}$ for $\mathrm{Ne}, 16.4 \mathrm{eV}$ for $\mathrm{Ar}$, and $14.1 \mathrm{eV}$ for $\mathrm{Kr}$. It decreases as the atomic number increases and exhibits the same tendency as in the case of the two-valence-electron systems. More-

TABLE III. The calculated DIE spectra of Ne, Ar, and $\mathrm{Kr}$ (in eV). The DIEs in the "GWA $\times 2$ " are twice the IPs within the GWA (which does not include the effect of the Coulomb repulsive interaction); " $T$ matrix" denotes the values evaluated by the ladder diagrams of the bare Coulomb interaction $U$, respectively. The assignments in the table are determined by the information of the eigenfunctions in Eq. (4).

\begin{tabular}{cccc}
\hline \hline Ne & GWA $\times 2$ & $T$ matrix & Experiment $^{\mathrm{a}}$ \\
\hline${ }^{3} \Pi$ & 44.56 & $\mathbf{6 8 . 8 0}$ & 62.52 \\
${ }^{1} \Delta$ & 44.56 & $\mathbf{7 1 . 6 5}$ & 65.73 \\
${ }^{1} \Sigma$ & 44.56 & $\mathbf{7 4 . 1 1}$ & 69.43 \\
\hline $\mathrm{Ar}$ & $\mathrm{GWA} \times 2$ & $T$ matrix & Experiment $^{\mathrm{a}}$ \\
\hline${ }^{3} \Pi$ & 31.82 & $\mathbf{4 5 . 5 0}$ & 43.38 \\
${ }^{1} \Delta$ & 31.82 & $\mathbf{4 7 . 3 3}$ & 45.11 \\
${ }^{1} \Sigma$ & 31.82 & $\mathbf{4 7 . 7 9}$ & 47.50 \\
\hline $\mathrm{Kr}$ & $\mathrm{GWA} \times 2$ & $T$ matrix & Experiment $^{\mathrm{a}}$ \\
\hline${ }^{3} \Pi$ & 28.16 & $\mathbf{3 9 . 7 8}$ & 38.36 \\
${ }^{1} \Delta$ & 28.16 & $\mathbf{4 1 . 4 1}$ & 40.18 \\
${ }^{1} \Sigma$ & 28.16 & $\mathbf{4 2 . 9 0}$ & 42.46 \\
\hline \hline
\end{tabular}

${ }^{\mathrm{a}}$ Reference 35 . 
TABLE IV. The calculated DIE spectra of the CO molecule (in eV). " $T$ matrix" denotes the values obtained by evaluating the ladder diagrams up to the infinite order of the bare Coulomb interactions and " $U_{\alpha \beta \gamma \delta}$ " denote the matrix element carrying the largest contribution to the DIE.

\begin{tabular}{ccccc}
\hline \hline & GWA $\times 2$ & $T$ matrix & $U_{\alpha \beta \gamma \delta}$ & Experiment $^{\mathrm{a}}$ \\
\hline $1{ }^{3} \Pi_{u}$ & 30.37 & 40.66 & 11.26 & 41.29 \\
$1{ }^{1} \Sigma_{g}^{+}$ & 27.82 & 40.79 & 14.98 & 41.70 \\
$1{ }^{1} \Pi_{g}$ & 30.37 & 41.61 & 11.26 & 41.81 \\
$1{ }^{3} \Sigma_{g}^{+}$ & 34.05 & 42.56 & 11.02 & 43.57 \\
$2{ }^{1} \Sigma_{g}^{+}$ & 34.05 & 46.83 & 11.02 & 45.48 \\
\hline \hline
\end{tabular}

${ }^{\mathrm{a}}$ Reference 37 .

over, we obtain that the effective Coulomb interaction, which can be expressed as $T$ matrix-GWA $\times 2$, is about $24.2 \mathrm{eV}$ for $\mathrm{Ne}, 13.7 \mathrm{eV}$ for $\mathrm{Ar}$, and $11.6 \mathrm{eV}$ for $\mathrm{Kr}$. The effective Coulomb interaction of the rare-gas atoms is different by $2.5-4.3 \mathrm{eV}$ from the on-site Coulomb interaction $U_{\alpha \alpha \alpha \alpha}$, namely, showing the importance of the ladder diagrams up to the infinite order.

The present results for Ne are good but not perfect. The reason is not clear and further investigation is required.

\section{C. $\mathrm{CO}$ and $\mathrm{C}_{2} \mathrm{H}_{2}$ molecules}

The IP of CO molecule evaluated using the LDA and the GWA is about 9.1 and $13.9 \mathrm{eV}$, respectively. The calculated IP within the GWA is in good agreement with the experiment $^{36}$ (The values for $\mathrm{CO}$ are already given in Ref. 32). In Table IV, the DIE spectra up to $47 \mathrm{eV}$ of the $\mathrm{CO}$ molecule are presented together with the corresponding experiments. The column of " $T$ matrix" in Table IV denotes the values obtained from the evaluation of the ladder diagrams up to the infinite order of the bare Coulomb interaction. The five DIEs in the table are characterized from lower to higher energies: $1{ }^{3} \Pi_{u}, 1{ }^{1} \Sigma_{g}^{+}, 1{ }^{1} \Pi_{g}, 1{ }^{3} \Sigma_{u}^{+}$, and $2{ }^{1} \Sigma_{g}^{+}$, where $1 \Pi$, $1 \Sigma$, and $2 \Sigma$ are the two-hole states composed mainly of $(1 \pi)(5 \sigma),(5 \sigma)^{2}$, and $(4 \sigma)(5 \sigma)$, respectively. Their characteristics are consistent with the experiment. Because "GWA $\times 2$ " does not include the effect of the Coulomb interactions between two holes, the values of "GWA $\times 2$ " are smaller than the corresponding experimental values by about $10 \mathrm{eV}$.
The ladder diagrams up to the infinite order considerably improve the results of "GWA $\times 2$." The present results are in good agreement with the experiment as well as previous calculations using the multireference configuration-interaction method. ${ }^{37}$

If we neglect the Coulomb interaction between two holes, $1{ }^{1} \Sigma_{g}^{+}$becomes the smallest DIE when two electrons are removed from the HOMO level. In reality, however, $1{ }^{3} \Pi_{u}$ becomes the smallest DIE when the electrons are individually removed from the HOMO and HOMO-1 because creating two holes in different orbitals can reduce the Coulomb interaction (see the column " $U_{\alpha \beta \gamma \delta}$ " in Table IV). Therefore, GWA $\times 2+U_{\alpha \beta \gamma \delta}$ results that $1 \Pi(41.6 \mathrm{eV})$ is smaller than $1 \Sigma(42.8 \mathrm{eV})$, although $1{ }^{3} \Pi_{g}$ and $1{ }^{1} \Pi_{g}$ (or $1{ }^{3} \Sigma_{g}^{+}$and $1{ }^{1} \Sigma_{g}^{+}$) are still same values. In order to distinguish the spin-triplet and singlet states of the created holes, we need to treat the off-diagonal elements of $H_{\alpha \beta \nu \mu}$ in Eq. (4). To diagonalize the full $H_{\alpha \beta \nu \mu}$ is equal to evaluate the ladder diagrams up to the infinite order.

Next, let us discuss the results of $\mathrm{C}_{2} \mathrm{H}_{2}$ molecule. The calculated IP within the LDA and the GWA is about 7.3 and $11.2 \mathrm{eV}$. The GWA considerably improves the result of the LDA. Five lower DIEs of $\mathrm{C}_{2} \mathrm{H}_{2}$ are presented in Table $\mathrm{V}$ together with the experiments ${ }^{38-40}$ and the results of the previous calculation based on the $\operatorname{ADC}(2) \operatorname{method}^{14}$ for comparison. The experimental values are measured by double charge transfer (DCT) between projectile $M^{+}(M=\mathrm{H}$ or $\mathrm{OH})$ and the target molecule. The DIEs of the molecule are uniquely determined by the conservation of energy and momentum in terms of the formation energy from $M^{+}$to $M^{-}$.

The characteristics determined by the calculation are consistent with the experiments, although ${ }^{1} \Delta$ and ${ }^{1} \Sigma$ are indistinguishable in the experimental $\mathrm{H}^{+} / \mathrm{DCT}$. The DIEs in the GWA $\times 2$ are again identical at ${ }^{3} \Sigma,{ }^{1} \Delta$, and ${ }^{1} \Sigma$ because of the twofold degeneracy of the $(1 \pi)$ level of $\mathrm{C}_{2} \mathrm{H}_{2}$. However, if we include the effect of the Coulomb interaction more accurately, ${ }^{3} \Sigma$ becomes the smallest. In other words, the Coulomb interaction can be further increased when two electrons are removed from the same orbital (HOMO or HOMO-1) rather than being individually removed from two different orbitals (HOMO and HOMO-1) (see Table V). If

TABLE V. The DIEs of $\mathrm{C}_{2} \mathrm{H}_{2}$ (in eV). " $T$ matrix" and " $U_{\alpha \beta \gamma \delta}$ " denote the results obtained by evaluating the ladder diagrams of the bare Coulomb interaction and the matrix element carrying the largest contribution to the DIE, respectively. The results of the $\mathrm{ADC}(2)$ and two kinds of experimental DCT results, $\mathrm{H}^{+} / \mathrm{DCT}$ and $\mathrm{OH}^{+} / \mathrm{DCT}$ corresponding to singlet and triplet states, respectively, are given for comparison.

\begin{tabular}{|c|c|c|c|c|c|c|}
\hline & \multicolumn{3}{|c|}{ Calculation } & \multicolumn{3}{|c|}{ Expt. } \\
\hline & $\mathrm{GWA} \times 2$ & $T$ matrix & $U_{\alpha \beta \gamma \delta}$ & $\operatorname{ADC}(2)^{\mathrm{a}}$ & $\mathrm{OH}^{+} / \mathrm{DCT}^{\mathrm{b}}$ & $\mathrm{H}^{+} / \mathrm{DCT}$ \\
\hline${ }^{3} \Sigma_{g}$ & 22.31 & 33.69 & 12.25 & 31.35 & $32.7 \pm 0.3$ & $\cdots$ \\
\hline${ }^{1} \Delta_{g}^{8}$ & 22.31 & 34.71 & 12.54 & 32.47 & $\cdots$ & $33.6 \pm 0.5,{ }^{\mathrm{c}} 33.7^{\mathrm{d}}$ \\
\hline${ }^{1} \Sigma_{g}^{8}$ & 22.31 & 35.36 & 12.25 & 33.24 & $\cdots$ & $33.6 \pm 0.5,^{\mathrm{c}} 33.7^{\mathrm{d}}$ \\
\hline${ }^{3} \Pi_{u}^{0}$ & 27.81 & 38.21 & 11.12 & 36.75 & $37.9 \pm 0.4$ & $\cdots$ \\
\hline${ }^{1} \Pi_{u}$ & 27.81 & 38.96 & 11.12 & 37.64 & $\cdots$ & $38.5 \pm 0.7^{\mathrm{c}} 38.4^{\mathrm{d}}$ \\
\hline
\end{tabular}


two electrons are removed from the same orbital, the DIEs split into ${ }^{3} \Sigma$ and ${ }^{1} \Sigma$ or ${ }^{3} \Pi$ and ${ }^{1} \Pi$ according to the symmetry of spin of the created holes. We confirmed that it is easier to remove two electrons in a triplet state ${ }^{3} \Sigma$ or ${ }^{3} \Pi$ than in a singlet state ${ }^{1} \Sigma$ or ${ }^{1} \Pi$. These results are consistent with the experiments or the results calculated by Ohrendorf et al. ${ }^{14}$

\section{SUMMARY}

In summary, we formulated the first-principles $T$-matrix theory starting from the GWA and evaluated the particleparticle ladder diagrams up to the infinite order by means of the BSE in order to calculate the DIE spectra. We firstly and successfully applied this theory to several atoms and small molecules and showed the importance of the ladder diagrams up to the infinite order. The effect of short-range electron correlation plays an important role in these spectra, particularly for atoms and small molecules. Our results are in excellent agreement with available experimental data as well as with the previous calculations.

These results show the significant effect of the electron correlations on the DIEs, i.e., the DIEs resulting from the present $T$-matrix theory are quite distinct from the simple sum of two one-particle energies obtained within the GWA. This $T$-matrix theory is advantageous in that it enables to calculate the spectra and wave functions of the two-particle excited states simultaneously and accurately. Moreover, this approach takes a moderate amount of the CPU time and computer memory. Currently, it is highly desirable to apply the present method to larger molecules to calculate their DIE spectra.

\section{ACKNOWLEDGMENT}

The authors are grateful to the Information Initiative Center, Hokkaido University, for the use of the HITACHI SR8000 supercomputing facilities.

${ }^{1}$ A. I. Boldyrev and J. Simons, J. Chem. Phys. 98, 4745 (1993).

${ }^{2}$ M. K. Scheller and L. S. Cederbaum, J. Chem. Phys. 99, 441 (1993).

${ }^{3}$ M. K. Scheller and L. S. Cederbaum, J. Chem. Phys. 100, 8934 (1994).

${ }^{4}$ J. Friedrich, P. Weis, J. Kaller, R. L. Whetten, and M. M. Kappes, Eur. Phys. J. D 9, 269 (1999).

${ }^{5}$ H. J. Werner and P. J. Knowles, J. Chem. Phys. 89, 5803 (1988).

${ }^{6}$ J. H. D. Eland, M. Hochlaf, G. C. King, P. S. Kreynin, R. J. Leroy, I. R. McNab, and J. M. Robbe, J. Phys. B 37, 3197 (2004).
${ }^{7}$ F. Tarantelli, A. Tarantelli, A. Sgamellotti, J. Schirmer, and L. S. Cederbaum, Chem. Phys. Lett. 117, 577 (1985).

${ }^{8}$ A. Tarantelli and L. S. Cederbaum, Phys. Rev. A 39, 1639 (1989).

${ }^{9}$ A. Tarantelli and L. S. Cederbaum, Phys. Rev. A 39, 1656 (1989).

${ }^{10}$ F. Tarantelli, A. Tarantelli, A. Sgamellotti, J. Schirmer, and L. S. Cederbaum, J. Chem. Phys. 83, 4683 (1985).

${ }^{11}$ F. Tarantelli, A. Sgamellotti, L. S. Cederbaum, and J. Schirmer, J. Chem. Phys. 86, 2201 (1987).

${ }^{12}$ J. Schirmer and G. Angonoa, J. Chem. Phys. 91, 1754 (1989).

${ }^{13}$ E. M. L. Ohrendorf, H. Köppel, L. S. Cederbaum, F. Tarantelli, and A. Sgamellotti, J. Chem. Phys. 91, 1734 (1989).

${ }^{14}$ E. M. L. Ohrendorf, F. Tarantelli, and L. S. Cederbaum, J. Chem. Phys. 92, 2984 (1990).

${ }^{15}$ M. S. Hybertsen and S. G. Louie, Phys. Rev. B 34, 5390 (1986).

${ }^{16}$ R. W. Godby, M. Schlüter, and L. J. Sham, Phys. Rev. B 35, 4170 (1987).

${ }^{17}$ L. Hedin, Phys. Rev. 139, A796 (1965).

${ }^{18}$ G. Onida, L. Reining, R. W. Godby, R. Del Sole, and W. Andreoni, Phys. Rev. Lett. 75, 818 (1995).

${ }^{19}$ G. Onida, L. Reining, and A. Rubio, Rev. Mod. Phys. 74, 601 (2002).

${ }^{20}$ S. Albrecht, R. Del Sole, and G. Onida, Phys. Rev. Lett. 80, 4510 (1998).

${ }^{21}$ M. Rohlfing and S. G. Louie, Phys. Rev. Lett. 80, 3320 (1998).

${ }^{22}$ M. Rohlfing and S. G. Louie, Phys. Rev. B 62, 4927 (2000).

${ }^{23}$ L. X. Benedict, E. L. Shirley, and R. B. Bohm, Phys. Rev. Lett. 80, 4514 (1998).

${ }^{24}$ M. Springer, F. Aryasetiawan, and K. Karlsson, Phys. Rev. Lett. 80, 2389 (1998).

${ }^{25}$ K. Ohno, F. Mauri, and S. G. Louie, Phys. Rev. B 56, 1009 (1997)

${ }^{26}$ S. Ishii, K. Ohno, Y. Kawazoe, and S. G. Louie, Phys. Rev. B 65, 245109 (2002).

${ }^{27}$ S. Ishii, K. Ohno, V. Kumar, and Y. Kawazoe, Phys. Rev. B 68, 195412 (2003).

${ }^{28}$ S. Ishii, K. Ohno, and Y. Kawazoe, Mater. Trans., JIM 42, 2150 (2001).

${ }^{29}$ J. Kanamori, Prog. Theor. Phys. 30, 275 (1963).

${ }^{30}$ U. C. Grossman, M. Rohlfing, L. Mitas, S. G. Louie, and M. L. Cohen, Phys. Rev. Lett. 86, 472 (2001).

${ }^{31}$ H. Yasuhara, Physica (Amsterdam) 78, 420 (1974).

${ }^{32}$ S. Ishii, K. Ohno, and Y. Kawazoe, Mater. Trans., JIM 45, 1411 (2004).

${ }^{33}$ C. Kittel, Introduction to Solid State Physics, 7th ed. (Wiley, New York, 1995).

${ }^{34}$ P. Erman, A. Karawajczyk, E. Rachlew-Källne, C. Strömholm, J. Larsson, A. Persson, and R. Zerne, Chem. Phys. Lett. 215, 173 (1993).

${ }^{35}$ K. M. McHugh, J. G. Eaton, G. H. Lee, H. W. Sarkas, L. H. Kidder, J. T. Snodgrass, M. R. Manaa, and K. H. Bowen, J. Chem. Phys. 91, 3792 (1989).

${ }^{36} \mathrm{http} / / /$ webbook.nist.gov/

${ }^{37}$ G. Dawber, A. G. McConkey, L. Avaldi, M. A. MacDonald, G. C. King, and R. I. Hall, J. Phys. B 27, 2191 (1994).

${ }^{38}$ S. R. Andrews, F. M. Harris, and D. E. Parry, Chem. Phys. 166, 69 (1992).

${ }^{39}$ J. Appell, J. Durup, F. C. Fehsenfeld, and P. Fournier, J. Phys. B: At. Mol. Phys. 7, 406 (1974).

${ }^{40}$ J. H. D. Eland, S. D. Price, J. G. Cheney, P. Lablanquie, I. Nenner, and P. G. Fournier, Philos. Trans. R. Soc. London, Ser. A 324, 247 (1988). 